\title{
Performance Monitoring of MPC Based on Dynamic Principal Component Analysis
}

\author{
Xue Min Tian* Gong Quan Chen** Yu Ping Cao* \\ Sheng Chen ${ }^{* * *}$ \\ * College of Information and Control Engineering, China University of \\ Petroleum, Dongying 257061, China (E-mails: tianxm@hdpu.edu.cn \\ caoypupc@gmail.com). \\ ** Automation Technique Center of China MCC20 Group Co., Ltd., \\ Shanghai201900, China (E-mail: chen9028@163.com). \\ *** School of Electronics and Computer Science, University of \\ Southampton, Southampton SO17 1BJ, United Kingdom (E-mail: \\ sqc@ecs.soton.ac.uk).
}

\begin{abstract}
A unified framework based on the dynamic principal component analysis (PCA) is proposed for performance monitoring of constrained multi-variable model predictive control (MPC) systems. In the proposed performance monitoring framework, the dynamic PCA based performance benchmark is adopted for performance assessment, while performance diagnosis is carried out using a unified weighted dynamic PCA similarity measure. Simulation results obtained from the case study of the Shell process demonstrate that the use of the dynamic PCA performance benchmark can detect the performance deterioration more quickly compared with the traditional PCA method, and the proposed unified weighted dynamic PCA similarity measure can correctly locate the root cause for poor performance of MPC controller.
\end{abstract}

Keywords: Model predictive control, performance monitoring, performance assessment, performance diagnosis, dynamic principal component analysis.

\section{INTRODUCTION}

The initial work on controller performance monitoring can be traced back to the research of Harris (1989), in which the minimum variance control (MVC) benchmark was proposed as the theoretical minimum variance bound. Since this ground-breaking study, much research has been focused on the performance assessment of both singleinput single-output (SISO) and multiple-input multipleoutput controllers as well as feedback, forward and cascade controllers, resulting in a large number of publications (Desborough and Harris, 1992; Harris et al., 1996, 1999; Harris and Seppala, 2002; Jelali, 2006; Qin and Yu, 2007; AlGhazzawi and Lennox, 2009) in addition to several commercial applications (Desborough and Miller, 2002; Gao et al., 2003). The increasing popularity of model predictive control (MPC) in industrial applications (Qin and Badgwell, 2003) has led to a high demand for the performance monitoring of MPC systems. Generally, performance monitoring includes performance assessment and diagnosis, and the task of MPC controller performance monitoring is composed of three steps (Harris et al., 1999):

(1) The determination of the proper benchmark which the assessed control system can be compared to.

(2) The establishment of proper monitoring statistics or performance indexes to assess the controller.

(3) The introduction of methods for diagnosing the root causes of performance degradation when the current poor performance of the MPC system is detected.
Although the research for the performance monitoring of MPC controllers is not studied as comprehensive as that for conventional feedback controllers, several academic studies and industrial applications have been reported, which mainly focus on the first two steps of performance monitoring, namely, performance assessment. In these studies, the most widely adopted method is the MVC benchmark. However, as the constraints and nonlinearity of the MPC algorithm make the theoretical MVC lower bound unrealisable, the MVC benchmark used for the performance monitoring of feedback controllers is not well suited to the performance monitoring of MPC systems.

Some alternative approaches have been adopted to evaluate MPC controllers. Shah et al. (2002) proposed the normalised multivariate impulse response (NMIR) plot which provides a graphical measure in terms of settling time, decay rates and other controller performance indicators, while Gao et al. (2003) applied this method to evaluate MPC controllers. However, this approach is based on deterministic performance assessment. Huang (1997) proposed to use the linear quadratic Gaussian (LQG) control as the benchmark for performance assessment, while Shah et al. (2002) applied this approach to MPC performance assessment. The main advantage of this approach is that the input variance is also taken into account, but it requires solving the complicated LQG problem. Zhao et al. (2009) applied the LQG benchmark to consider the economic aspects in the procedure of performance assessment. Multivariate statistic methods, such as prin- 
cipal component analysis (PCA) and partial least square (PLS), were introduced to controller performance assessment (Zhang and Li, 2006; AlGhazzawi and Lennox, 2009). Patwardhan et al. (2002) proposed a performance index based on the comparison of the designed and achieved objective functions. Similarly, Schäfer and Cinar (2004) proposed two performance indexes, which use the ratio of historical and achieved performance for assessment and the ratio of designed and achieved performance for diagnosis. However, the ratio of designed and achieved performance can only distinguish two classes of performance deterioration, namely, those problems associated with the controller and those problems not caused by the controller.

There also exist some other performance monitoring approaches to evaluate the MPC controller performance directly without using any performance index, which mainly focus on the performance diagnosis aspect. In the works (Loquasto and Seborg, 2003b,a), neural network-based and PCA similarity based pattern classifiers were utilised to classify the MPC performance into four classes in order to accomplish diagnosis procedure. Patwardhan and Shah (2002) quantified the effect of constraints, modeling uncertainty, disturbance uncertainty and process nonlinearity on the closed loop performance. Engineers may use the contribution of these factors on the performance to diagnose and to locate the corresponding causes of performance degradation. The disadvantage of the above methods is that the performance status could not be estimated from a quantitative index. From a consideration of set-point tracking, Sotomayor and Odloak (2006) compared the performance of MPC controller in operation with another ideal controller which inherits the structure, input constraints and tuning parameters of the controller been evaluated. But it is complicated to build such an ideal controller. Thornhill and Horch (2006) discussed the causes of plant-wide disturbances which may influence the performance of controllers, while Zhao et al. (2008) took the predictive error into the consideration in the performance assessment. Julien et al. (2004) presented a SISO MPC performance curve created by plotting the variance of the controlled variable against that of the differential manipulated variable over a range of values for the socalled move suppression parameter. By analysing this performance curve, it can be determined whether the poor performance is caused by the controller itself or by the plant model. Yu and Qin (2008) introduced the loadingbased and angle-based contributions into the performance diagnosis of MPC systems, which focused on the variable identification. Huang (2008) established a probabilistic framework using the Bayesian method to analyse whether the four components of the control loop each works at a satisfied status. This approach only considered the controller performance diagnosis, and no quantitative index was given to detect controller performance deterioration.

Against this background, we propose a multi-variable MPC performance monitoring framework, which includes all the three steps of monitoring procedure. Specifically, a performance benchmark is established using the statistical thresholds which capture the covariance information of the principal components as well as the residual subspace, and the performance indexes based on the dynamic PCA analysis are introduced to evaluate the MPC controller.
Then, a performance diagnosis approach based on the unified weighted dynamic PCA similarity measure is proposed, which measures the similarity degrees between the current data and the predefined data classes and isolates the root cause of the performance deterioration according to the maximum similarity. A case study involving the Shell process is used to demonstrate the effectiveness of the proposed performance monitoring procedure. The reminder of this paper is organized as follows. A performance assessment based on the dynamic PCA benchmark is introduced in Section 2, while the performance diagnosis technique using pattern classification according to the similarity measure is proposed in Section 3. The proposed performance monitoring procedure is then summarised in Section 4 . Section 5 provides a case study to illustrate the effectiveness of the proposed performance monitoring framework, and our conclusions are offered in Section 5.

\section{PERFORMANCE ASSESSMENT USING DYNAMIC PCA}

MPC computes the future control actions by minimizing an objective function over a finite prediction horizon according to the historical information and future input of the process model. Usually, the cost function is related to the covariance matrix of the output variables $\mathbf{y}(k)=\left[y_{1}(k) y_{2}(k) \cdots y_{n}(k)\right]^{\mathrm{T}}$ and the covariance matrix of the input variables $\mathbf{u}(k)=\left[u_{1}(k) u_{2}(k) \cdots u_{m}(k)\right]^{T}$. Furthermore, the model predictive error vector $\mathbf{e}(k)=$ $\left[e_{1}(k) e_{2}(k) \cdots e_{n}(k)\right]^{T}$ is affected by the control action and the level of process-model mismatch as well as the plant disturbances. Therefore, the model predictive errors, controlled variables and control variables should be included into the following monitoring variable set

$\mathbf{x}(k)=\left[u_{1}(k) \cdots u_{m}(k) e_{1}(k) \cdots e_{n}(k) y_{1}(k) \cdots y_{n}(k)\right]^{\mathrm{T}} \cdot(1)$

The dimension of $\mathbf{x}(k)$ is $\bar{m}=2 n+m$.

In order to maximise the information of covariance, singular value decomposition (SVD) is carried out in PCA $(\mathrm{Ku}$ et al., 1995). Give a training data set of $\bar{n}$ observations at the time $k$

$$
\mathbf{X}_{0}=\mathbf{X}_{0}(k)=\left[\begin{array}{c}
\mathbf{x}^{\mathrm{T}}(k) \\
\mathbf{x}^{\mathrm{T}}(k-1) \\
\vdots \\
\mathbf{x}^{\mathrm{T}}(k-\bar{n}+1)
\end{array}\right] .
$$

The loading matrix $\mathbf{P} \in \mathrm{R}^{\bar{m} \times a}$ with the $a$ loading vectors can be obtained via the SVD of $\mathbf{X}_{0}$. Given a new observation vector $\mathbf{x}_{\text {new }}$, the principal components $\mathbf{t}$ and the residual variables $\mathbf{r}$ can be obtained as follows

$$
\begin{aligned}
& \mathbf{t}=\mathbf{P}^{\mathrm{T}} \mathbf{x}_{\text {new }}, \\
& \mathbf{r}=\left(\mathbf{I}-\mathbf{P} \mathbf{P}^{\mathrm{T}}\right) \mathbf{x}_{\text {new }},
\end{aligned}
$$

where $\mathbf{I}$ denotes the identity matrix of appropriate dimension. The two statistics, $T^{2}$ and SPE, are defined by

$$
\begin{aligned}
T^{2} & =\mathbf{t}^{\mathrm{T}} \boldsymbol{\Sigma}^{-2} \mathbf{t}, \\
\mathrm{SPE} & =\mathbf{r}^{\mathrm{T}} \mathbf{r},
\end{aligned}
$$

where $\boldsymbol{\Sigma}=\operatorname{diag}\left\{\sigma_{1}, \sigma_{2}, \cdots, \sigma_{a}\right\}$ with $\sigma_{j}, 1 \leq j \leq a$, being the first $a$ singular values of $\mathbf{X}_{0}$. The two thresholds for $T^{2}$ and SPE can be calculated as follows 


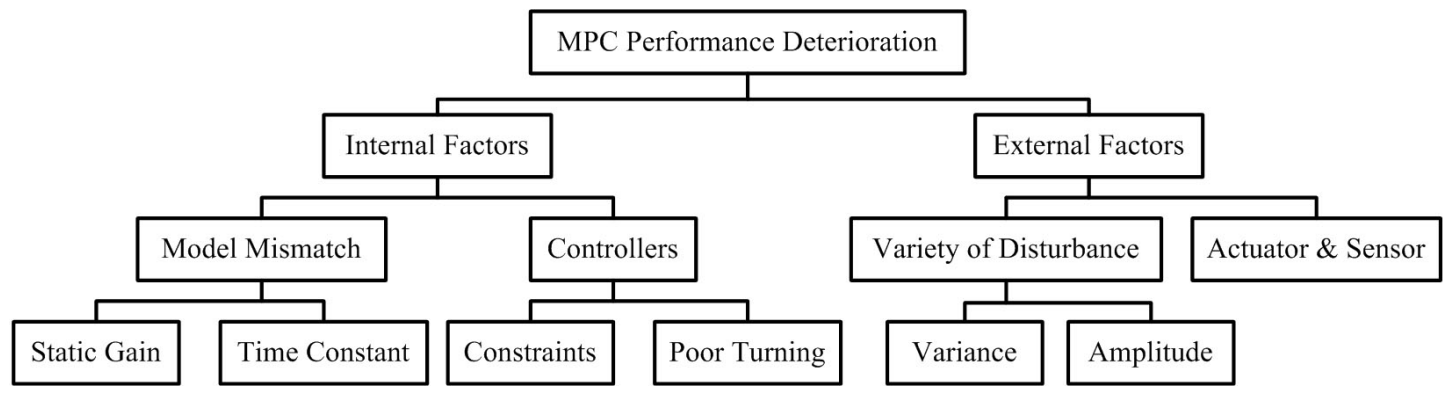

Fig. 1. Illustrative schematic of performance deterioration causes.

$$
\begin{aligned}
T_{\alpha}^{2} & =\frac{a(\bar{n}-1)(\bar{n}+1)}{\bar{n}(\bar{n}-a)} F_{\alpha}(a, \bar{n}-a), \\
\mathrm{SPE}_{\alpha} & =\phi_{1}\left(\frac{h_{0} c_{\alpha} \sqrt{2 \phi_{2}}}{\phi_{1}}+1+\frac{\phi_{2} h_{0}\left(h_{0}-1\right)}{\phi_{1}^{2}}\right),
\end{aligned}
$$

where $F_{\alpha}(a, \bar{n}-a)$ denotes the $F$-distribution with the confidence level $\alpha$ and the freedom level of $a$ and $\bar{n}-a$,

$$
\phi_{i}=\sum_{j=a+1}^{\bar{n}} \sigma_{j}^{2 i}
$$

$h_{0}=1-2 \phi_{1} \phi_{3} /\left(3 \phi_{2}^{2}\right), c_{\alpha}$ denotes the confidence limit $(1-\alpha) \%$ of the Gaussian distribution, and $\sigma_{j}$ denotes the $j$ th singular value of $\mathbf{X}_{0}$.

For dynamic systems, not only the correlation of the process variables but also the correlation of the dynamic time series should be taken into account. That is, the relationship between $\mathbf{X}_{0}(k)$ and $\mathbf{X}_{0}\left(k-k_{s}\right)$ should be considered in performing PCA, where $k_{s}$ denotes the time lag which is appropriately chosen to maximise the influence of the data. Extending the training data to the previous $k_{s}$ steps leads to the augmented data set

$$
\overline{\mathbf{X}}_{0}=\left[\mathbf{X}_{0}(k) \mathbf{X}_{0}(k-1) \cdots \mathbf{X}_{0}\left(k-k_{s}\right)\right] \text {. }
$$

The previous PCA analysis can readily be applied to the augmented training data set $\overline{\mathbf{X}}_{0}$, which we refer to as the dynamic PCA (DPCA). By contrast, the traditional PCA is based on analysing $\mathbf{X}_{0}(k)$ only.

A period of the reference data is chosen as the user-defined benchmark, which generally should be a period of the "gold" operation data from the process with a satisfactory control performance. Let the benchmark period be denoted by $I$ and the monitored period by $I I$. Further denote the reference data as $\overline{\mathbf{X}}_{I}$ and the data of the monitored period by $\overline{\mathbf{X}}_{I I}$. The DPCA is carried out on $\overline{\mathbf{X}}_{I}$, and the two thresholds for the statistics $T^{2}$ and SPE, denoted as denoted as $L_{I}^{T^{2}}$ and $L_{I}^{\mathrm{SPE}}$, respectively, are obtained according to (7) and (8). $L_{I}^{T^{2}}$ and $L_{I}^{\mathrm{SPE}}$ are used as the performance benchmark. To assess the monitored data at the time $k$, the statistics of the monitored data $\overline{\mathbf{X}}_{I I}$, denoted as $T_{I I}^{2}(k)$ and $\operatorname{SPE}_{I I}(k)$, are then calculated according to (5) and (6). The performance indexes for assessing the MPC controller at the time $k$ of the period II are then defined as follows

$$
\begin{aligned}
\eta_{T^{2}}(k) & =\frac{L_{I}^{T^{2}}}{T_{I I}^{2}(k)}, \\
\eta_{\mathrm{SPE}}(k) & =\frac{L_{I}^{\mathrm{SPE}}}{\operatorname{SPE}_{I I}(k)} .
\end{aligned}
$$

\section{PERFORMANCE DIAGNOSIS USING UNIFIED WEIGHTED DYNAMIC PCA SIMILARITY}

Generally, there exist many causes that could lead to a poor performance of a MPC controller. When the performance indexes indicate that the current performance of the controller is not satisfactory, a diagnosis procedure should be invoked to locate the root cause of this performance degradation. The main causes for MPC performance deterioration are shown in Fig. 1. We propose a similarity measure based classification method to realize the performance diagnosis by comparing the similarities between the current monitored data with the pre-defined data classes. The maximum similarity indicates which class or cause of poor performance that the current data belongs to.

The PCA similarity measure $S_{\mathrm{PCA}}$ defined in

$$
S_{\mathrm{PCA}}=\frac{1}{a} \sum_{i=1}^{a} \sum_{j=1}^{a} \cos ^{2} \theta_{i j}=\frac{1}{a} \operatorname{tr}\left\{\mathbf{C}_{1}^{\mathrm{T}} \mathbf{C}_{2} \mathbf{C}_{2}^{\mathrm{T}} \mathbf{C}_{1}\right\},
$$

describes the degree of similarity between the two data sets $\mathbf{X}_{1}$ and $\mathbf{X}_{2}$, where $\operatorname{tr}\{\}$ denotes the matrix trace operator, $\mathbf{C}_{1}$ and $\mathbf{C}_{2}$ are the principal component subspaces corresponding to the two data sets, $a$ is the number of principal components, while $\theta_{i j}$ is the angle between the $i$ th principal component of $\mathbf{C}_{1}$ and the $j$ th principal component of $\mathbf{C}_{2}$.

Let $\boldsymbol{\Gamma}_{i}=\operatorname{diag}\left\{\sqrt{\lambda_{1}^{(i)}}, \sqrt{\lambda_{2}^{(i)}}, \cdots, \sqrt{\lambda_{a}^{(i)}}\right\}$ with $\lambda_{j}^{(i)}, 1 \leq j \leq$ $a$, being the first $a$ eigenvalues of $\mathbf{X}_{i}^{\mathrm{T}} \mathbf{X}_{i}$ for $i=1,2$. The weighted PCA (WPCA) similarity measure is defined as

$$
S_{\mathrm{PCA}}^{\lambda}=\frac{\operatorname{tr}\left\{\tilde{\mathbf{C}}_{1}^{\mathrm{T}} \tilde{\mathbf{C}}_{2} \tilde{\mathbf{C}}_{2}^{\mathrm{T}} \tilde{\mathbf{C}}_{1}\right\}}{\sum_{i=1}^{a} \lambda_{i}^{(1)} \lambda_{i}^{(2)}},
$$

where $\tilde{\mathbf{C}}_{i}=\mathbf{C}_{i} \boldsymbol{\Gamma}_{i}$ for $i=1,2$. From the definition (13), it can be seen that the more consistent the two data sets are in the principal component subspaces, the closer to 1 the WPCA similarity measure $S_{\mathrm{PCA}}^{\lambda}$ is.

If the DPCA is applied to the two augmented data sets $\overline{\mathbf{X}}_{1}$ and $\overline{\mathbf{X}}_{2}$, we obtain the weighted DPCA (WDPCA) similarity measure $S_{\mathrm{DPCA}-\mathrm{PCS}}^{\lambda}$ for the corresponding two dynamic principal component subspaces, which is given by

$$
S_{\mathrm{DPCA}-\mathrm{PCS}}^{\lambda}=\frac{\operatorname{tr}\left\{\tilde{\mathbf{C}}_{1}^{\mathrm{T}} \tilde{\mathbf{C}}_{2} \tilde{\mathbf{C}}_{2}^{\mathrm{T}} \tilde{\mathbf{C}}_{1}\right\}}{\sum_{i=1}^{a} \lambda_{i}^{(1)} \lambda_{i}^{(2)}},
$$


where the two principal component subspaces are obtained by the DPCA. We are now ready to introduce the proposed unified-weighted DPCA (UWDPCA) similarity measure. In the traditional process fault detection, the principal component subspace is used to reflect the main changes of process status or system. However, there is some valuable information, such as noises and unmeasured disturbances that are not presented in the principal component subspace but are included in the residual subspace (Yue and Qin, 2001; Alcala and Qin, 2009). Therefore, not only the WDPCA similarity measure of the two principal component subspaces, $S_{\mathrm{DPCA}-\mathrm{PCS}}^{\lambda}$ of (14), but also the similarity measure of the two residual subspaces, $S_{\mathrm{DPCA}-\mathrm{RS}}^{\lambda}$, should be considered. The expression of $S_{\mathrm{DPCA}-\mathrm{RS}}^{\lambda}$ is given as follows

$$
S_{\mathrm{DPCA}-\mathrm{RS}}^{\lambda}=\frac{\operatorname{tr}\left\{\tilde{\mathbf{G}}_{1}^{\mathrm{T}} \tilde{\mathbf{G}}_{2} \tilde{\mathbf{G}}_{2}^{\mathrm{T}} \tilde{\mathbf{G}}_{1}\right\}}{\sum_{i=1}^{a} \lambda_{i}^{(1)} \lambda_{i}^{(2)}},
$$

where $\tilde{\mathbf{G}}_{i}=\mathbf{G}_{i} \boldsymbol{\Gamma}_{i}$ for $i=1,2$ are the the two weighted residual subspaces, while $\mathbf{G}_{i}$ for $i=1,2$ are the two residual subspaces, related to the two data sets. The UWDPCA similarity measure is then defined

$$
S_{\mathrm{DPCA}}^{\lambda}=\beta \cdot S_{\mathrm{DPCA}-\mathrm{PCS}}^{\lambda}+(1-\beta) \cdot S_{\mathrm{DPCA}-\mathrm{RS}}^{\lambda},
$$

where the weighting factor, $\beta$, and should appropriately be selected according to the specified monitored process.

\section{PERFORMANCE MONITORING PROCEDURE}

The proposed DPCA-based MPC controller performance monitoring procedure can now be described as follows.

1) Let the number of performance classes be $K$, and suppose that the training data sets $\overline{\mathbf{X}}_{i}$ correspond to the classes $C_{i}$ for $1 \leq i \leq K$ are available. Further assume that the subspace of each class is established and stored in the database of performance patterns.

2) According to the experience or prior knowledge, a period of the process data, $\overline{\mathbf{X}}_{I}$, which meets the requirements of the designed performance, is selected as the benchmark data. The DPCA modeling is carried out on $\overline{\mathbf{X}}_{I}$, and the two thresholds, $L_{I}^{T^{2}}$ and $L_{I}^{\mathrm{SPE}}$, are obtained which correspond to the two statistics, $T^{2}$ and SPE, for $\overline{\mathbf{X}}_{I}$. The two thresholds are used as the performance benchmark for MPC controller performance assessment.

3) From the current monitored data $\overline{\mathbf{X}}_{I I}$, the two statistics, $T^{2}$ and SPE, are obtained, and the DPCA based performance indexes are calculated using (10) and (11).

4) If $\eta_{T^{2}}(k)$ and $\eta_{\mathrm{SPE}}(k)$ are greater or equal to 1 , the current controller performance is satisfactory or even better than the performance of the benchmark data. Then go to step 3 )

If any of $\eta_{T^{2}}(k)$ and $\eta_{\mathrm{SPE}}(k)$ is smaller than 1 , it is considered that the current controller performance has deteriorated. Then go to step 5).

5) If a poor performance is detected, the current data $\overline{\mathbf{X}}_{I I}$ of the control system is extracted to established its performance subspace.
6) Calculate the UWDPCA similarity $S_{i}\left(\overline{\mathbf{X}}_{i}, \overline{\mathbf{X}}_{I I}\right)$ between the current data $\overline{\mathbf{X}}_{I I}$ and each data set $\overline{\mathbf{X}}_{i}$ of the performance pattern database according to (16), where $1 \leq i \leq K$. Define

$$
i_{\max }=\arg \max _{1 \leq i \leq K} S_{i}\left(\overline{\mathbf{X}}_{i}, \overline{\mathbf{X}}_{I I}\right),
$$

and compute the scaled UWDPCA similarity measures

$$
\zeta_{j}=S_{j}\left(\overline{\mathbf{X}}_{j}, \overline{\mathbf{X}}_{I I}\right) / S_{i_{\max }}\left(\overline{\mathbf{X}}_{i_{\max }}, \overline{\mathbf{X}}_{I I}\right), 1 \leq j \leq K .(18)
$$

The classification rule is then given by

$$
C_{I I} \subset C_{j_{\max }} \text { if } j_{\max }=\arg \max _{1 \leq j \leq K} \zeta_{j} .
$$

That is, the current controller performance can be classified as the $C_{j_{\max }}$ class or $C_{j_{\max }}$ is the root cause of the controller performance deterioration.

\section{CASE STUDY}

\subsection{Shell Distillation Model}

The Shell tower, which is the main part of heavy oil catalytic cracking unit, is a typical multi-variable constrained process that has been used in many previous investigations of process control, monitoring and identification (Sotomayor and Odloak, 2006). The transfer function of this distillation column model is given by

$$
\begin{aligned}
{\left[\begin{array}{l}
y_{1}(s) \\
y_{2}(s) \\
y_{3}(s)
\end{array}\right]=} & {\left[\begin{array}{ccc}
\frac{4.05 e^{-27 s}}{50 s+1} & \frac{1.77 e^{-28 s}}{60 s+1} & \frac{5.88 e^{-27 s}}{50 s+1} \\
\frac{5.39 e^{-18 s}}{50 s+1} & \frac{5.72 e^{-14 s}}{60 s+1} & \frac{6.90 e^{-15 s}}{40 s+1} \\
\frac{4.38 e^{-20 s}}{33 s+1} & \frac{4.42 e^{-22 s}}{44 s+1} & \frac{7.20}{19 s+1}
\end{array}\right]\left[\begin{array}{l}
u_{1}(s) \\
u_{2}(s) \\
u_{3}(s)
\end{array}\right] } \\
& +\left[\begin{array}{cc}
\frac{3.60 e^{-27 s}}{45 s+1} & \frac{1.44 e^{-27 s}}{40 s+1} \\
\frac{1.52 e^{-15 s}}{25 s+1} & \frac{1.83 e^{-15 s}}{20 s+1} \\
\frac{1.14}{27 s+1} & \frac{1.26 e^{-5 s}}{32 s+1}
\end{array}\right]
\end{aligned}
$$

where $y_{i}$ and $u_{i}, 1 \leq i \leq 3$, are the controlled variables and manipulating variables, respectively, while $d_{1}$ and $d_{2}$ are the zero-mean Gaussian distributed disturbance variables.

The closed-loop control simulation was performed using a constrained MPC strategy. The sampling time was set to 1 minute. High and low constraints as well as saturation limits were imposed on the inputs, outputs and input increment velocities. The benchmark parameters of the MPC controller were tuned by trial-and-error to yield a reasonably satisfactory control performance. The model time horizon was set to 80 . The unmeasured disturbances $d_{1}$ and $d_{2}$ with the variance 0.01 were added to the simulation but there was no model-mismatch in generating the benchmark data. The set points of the controlled variables, $y_{1}$ and $y_{2}$, were all set to 1 , while the set point of $y_{3}$ was set to zero. The prediction horizon and the control horizon were tuned to be 20 and 3, respectively. Considering the settling time of the control system, each simulation window was set to 1000 minutes to allow the plant reached the steady state. 
Table 1. Classes of performance deterioration and related parameter values in generating the training data.

\begin{tabular}{c|c|c|c}
\hline \hline Class & $\begin{array}{c}\text { Operation } \\
\text { condition }\end{array}$ & $\begin{array}{c}\text { Related } \\
\text { parameter }\end{array}$ & Value/range \\
\hline$C_{1}$ & Disturbance & Mean & +0.2 \\
\hline$C_{2}$ & Model mismatch & $\begin{array}{c}\text { Gains } \\
\text { of first column }\end{array}$ & $\times 2.0$ \\
\hline$C_{3}$ & Model mismatch & $\begin{array}{c}\text { Time constant } \\
\text { of first column }\end{array}$ & $\times 2.0$ \\
\hline$C_{4}$ & $\begin{array}{c}\text { Constraint/ } \\
\text { Saturation }\end{array}$ & $\begin{array}{c}\text { Constraint } \\
\text { of outputs }\end{array}$ & $(-0.7,0.7)$ \\
\hline$C_{5}$ & Disturbance & Variance & 0.02 \\
\hline \hline
\end{tabular}

\subsection{Experimental Set-up}

A period of the reference data was chosen as the userspecified benchmark based on the well-tuned MPC controller mentioned in the previous subsection. Moreover, five prior-known causes to the performance deterioration of the distillation control system were established in Table 1. The five causes of the controller performance deterioration were the mean perturbation of disturbance $\left(C_{1}\right)$, the gain changes of the first column of the process model $\left(C_{2}\right)$, the time constant changes of the first column of the process model $\left(C_{3}\right)$, and the constraint saturation of the outputs of the MPC controller $\left(C_{4}\right)$ as well as the variance perturbation of the unmeasured disturbance $\left(C_{5}\right)$. More specifically, in generating the training data, the value of the disturbance mean in the $C_{1}$ period was increased by 0.2 . As to the $C_{2}$ period, the gains of the first column of the transfer function model were set to be two times of the nominal ones, while the time constants of the first column of the transfer function model in the $C_{3}$ period was set to two times of the nominal values. The controlled variable constraints in the $C_{4}$ period were set to be between -0.7 and 0.7 , which made the set point for $y_{1}$ and $y_{2}$ unreachable, while the constraints on the manipulating variables were specified in the range of -0.2 to 0.2 and the constraint on the input increment was between -0.05 and 0.05 . The value of the disturbance variance in the $C_{5}$ period was set to 0.02 , in comparison to the value of 0.01 in the reference period. In our simulation, 1000 samples were generated in the benchmark and in each of the five training periods.

\subsection{Experimental Results}

The first three classes of performance deterioration were simulated with the ramp changes occurred at the time $k=300$ minutes. The two assessment methods, the PCA and DPCA, were carried out on the data, and the detection times of performance deterioration for these three classes are shown in Table 2. From Table 2, it can can be observed that the DPCA based performance assessment method

Table 2. Comparison of detection time for the PCA and DPCA based performance assessment methods.

\begin{tabular}{c|c|c|c|c}
\hline \multirow{2}{*}{ Class } & \multicolumn{2}{|c|}{ PCA } & \multicolumn{2}{c}{ DPCA } \\
\cline { 2 - 5 } & SPE & $T^{2}$ & SPE & $T^{2}$ \\
\hline$C_{1}$ & 340 & 312 & 322 & 312 \\
\hline$C_{2}$ & 315 & 316 & 313 & 314 \\
\hline$C_{3}$ & 338 & 336 & 330 & 333 \\
\hline
\end{tabular}

Table 3. Performance diagnosis results for the FP1 period.

\begin{tabular}{c|c|c|c|c|c}
\hline Measure & $C_{1}$ & $C_{2}$ & $C_{3}$ & $C_{4}$ & $C_{5}$ \\
\hline WPCA & 0.9621 & 1.0000 & 0.4490 & 0.3108 & 0.4876 \\
\hline WDPCA & 0.9621 & 1.0000 & 0.4488 & 0.3107 & 0.4874 \\
\hline UWDPCA & 1.0000 & 0.8851 & 0.4517 & 0.4922 & 0.6411 \\
\hline
\end{tabular}

detected the performance deterioration earlier than the traditional PCA based method. For example, with the $T^{2}$ index, the DPCA based method detected the $C_{2}$ and $C_{3}$ classes of performance deterioration 2 and 3 minutes earlier than the PCA based method, respectively.

The proposed UWDPCA similarity measure of (16) was then applied to the performance diagnosis of the Shell process, and the weighting factor was set empirically to $\beta=0.6$. As a comparison, the traditional WPCA similarity measure of (13) and the WDPCA similarity measure of (14) were also used.

First, one period of the monitored data, denoted as the FP1, were generated by setting the disturbance mean to 0.36. Obviously, the FP1 data belongs to the $C_{1}$ class of performance deterioration. Table 3 shows the performance diagnosis results on the FP1 data using the WPCA, WDPCA and UWDPCA methods, where the the corresponding normalised similarity measure values $\zeta_{j}$, $1 \leq j \leq 5$, are given. It can be seen from Table 3 that the WPCA and WDPCA similarity measures could not locate the root cause of performance deterioration, while the UWDPCA similarity measure correctly identified that the $C_{1}$ class was the root cause of poor performance. This demonstrates the enhanced discriminant power of the UWDPCA similarity measure by including the residual subspace information.

Another period of the monitored data, denoted as the FP2, were then produced by changing the static gains in the first column of the transfer function model to 1.4 times of their nominal values. Table 4 shows the performance diagnosis results on the FP2 data using the WPCA, WDPCA and UWDPCA methods. In this case, all the three methods correctly indicated the $C_{1}$ class as the root cause of performance deterioration. The UWDPCA similarity measure again demonstrated a stronger discriminant power and could distinguish the correct root cause from other influence factors better. For example, the second highest similarity value was $\zeta_{1}=0.8322$ for the WPCA, $\zeta_{1}=0.6718$ for the WDPCA, and $\zeta_{1}=0.5509$ for the UWDPCA.

The third period of monitored data, denoted as the FP3, were generated by changing the time constants of the first column of the transfer function to 2.6 times of their nominal values. The performance diagnosis results given in Table 5 demonstrate that the three methods all correctly indicated that $C_{3}$ class was the root cause of performance deterioration. Moreover, the UWDPCA similarity measure

Table 4. Performance diagnosis results for the FP2 period.

\begin{tabular}{c|c|c|c|c|c}
\hline Measure & $C_{1}$ & $C_{2}$ & $C_{3}$ & $C_{4}$ & $C_{5}$ \\
\hline WPCA & 0.8322 & 1.0000 & 0.2965 & 0.2031 & 0.3347 \\
\hline WDPCA & 0.6718 & 1.0000 & 0.0526 & 0.0581 & 0.0767 \\
\hline UWDPCA & 0.5509 & 1.0000 & 0.0254 & 0.3183 & 0.3329 \\
\hline
\end{tabular}


Table 5. Performance diagnosis results for the FP3 period.

\begin{tabular}{c|c|c|c|c|c}
\hline Measure & $C_{1}$ & $C_{2}$ & $C_{3}$ & $C_{4}$ & $C_{5}$ \\
\hline WPCA & 0.4908 & 0.4964 & 1.0000 & 0.5890 & 0.8831 \\
\hline WDPCA & 0.1240 & 0.0068 & 1.0000 & 0.4189 & 0.9248 \\
\hline UWDPCA & 0.1307 & 0.0094 & 1.0000 & 0.6320 & 0.8981 \\
\hline
\end{tabular}

could distinguish the correct $C_{3}$ root cause from the other $C_{5}$ influence factor better than the WDPCA one.

\section{CONCLUSIONS}

We have proposed a unified framework based on the dynamic PCA for the performance monitoring of constrained multi-variable MPC systems. Specifically, the dynamic PCA based performance benchmark is adopted to assess the performance of a MPC controller. Both the dynamic principal component subspace and the residual subspace are extracted to establish the unified weighted similarity measure for measuring the degree of similarity between the current monitored data and a predefined data class. The root cause of performance deterioration can then be located by pattern classification according to the maximum similarity. A case study involving the Shell process has demonstrated the effectiveness of the proposed MPC performance assessment and diagnosis framework.

\section{REFERENCES}

Alcala, C.F. and Qin, S.J. (2009). Reconstruction-based contribution for process monitoring. Automatica, 45(7), 1593-1600.

AlGhazzawi, A. and Lennox, B. (2009). Model predictive control monitoring using multivariate statistics. $J$ Process Control, 19(2), 314-327.

Desborough, L. and Harris, T.J. (1992). Performance assessment measures for univariate feedback control. Canadian J. Chemical Eng., 70(6), 1186-1197.

Desborough, L. and Miller, R. (2002). Increasing customer value of industrial control performance monitoring honeywells experience. In AICHE Symposium Series, volume 326, 169-189.

Gao, J.P., Patwardhan, R., Akamatsu, K., Hashimoto, Y., Emoto, G., Shah, S.L., and Huang, B. (2003). Performance evaluation of two industrial mpc controllers. Control Engineering Practice, 11(12), 1371-1387.

Harris, T.J. (1989). Assessment of closed-loop performance. Canadian J. Chemical Eng., 67(10), 856-861.

Harris, T.J., Boudreau, F., and Macgregor, J.F. (1996). Performance assessment of multivariable feedback controllers. Automatica, 32(11), 1505-1518.

Harris, T.J. and Seppala, C.T. (2002). Recent developments in controller performance monitoring and assessment techniques. In AICHE Symposium Series, volume 326, 208-222. Tuscon, Arizona.

Harris, T.J., Seppala, C.T., and Desborough, D. (1999). A review of performance monitoring and assessment techniques for univariate and multivariate control systems. J. Process Control, 9(1), 1-17.

Huang, B. (1997). Multivariate Statistical Methods for Control Loop Performance Assessment. Ph.D. thesis, Dept. Chemical Eng., University of Alberta, Canada.

Huang, B. (2008). Bayesian methods for control loop monitoring and diagnosis. J. Process Control, 18(9), $829-838$
Jelali, M. (2006). An overview of control performance assessment technology and industrial applications. Control Engineering Practice, 14(5), 441-466.

Julien, R.H., Foley, M.W., and Cluett, W.R. (2004). Performance assessment using a model predictive control benchmark. J. Process Control, 14(4), 441-456.

$\mathrm{Ku}, \mathrm{W}$. , Storer, R.H., and Gergakis, C. (1995). Disturbance detection and isolation by dynamic principal component analysis. Chemometrics and Intelligent Laboratory Systems, 30(1), 179-196.

Loquasto, F. and Seborg, D.E. (2003a). Model predictive controller monitoring based on pattern classification and pca. In Proc. 2003 American Control Conference, volume 3, 1968-1973. Denver, Colorado.

Loquasto, F. and Seborg, D.E. (2003b). Monitoring model predictive control systems using pattern classification and neural networks. Industrial \& Engineering Chemistry Research, 42(20), 4689-4701.

Patwardhan, R.S. and Shah, S.L. (2002). Issues in performance diagnostics of model-based controllers. J. Process Control, 12(3), 413-427.

Patwardhan, R.S., Shah, S.L., and Qi, K.Z. (2002). Assessing the performance of model predictive controllers. Canadian J. Chemical Engineering, 80(5), 954-966.

Qin, S.J. and Badgwell, T.A. (2003). A survey of industrial model predictive control technology. Control Engineering Practice, 11(7), 733-764.

Qin, S.J. and Yu, J. (2007). Recent developments in multivariable controller performance monitoring. $J$. Process Control, 17(3), 221-227.

Schäfer, J. and Cinar, A. (2004). Multivariable mpc system performance assessment, monitoring, and diagnosis. $J$. Process Control, 14(2), 113-129.

Shah, S.L., Patwardhan, R., and Huang, B. (2002). Multivariate controller performance analysis: methods, applications and challenges. In AICHE Symposium Series, volume 326, 190-207.

Sotomayor, O.A.Z. and Odloak, D. (2006). Performance assessment of model predictive control systems. In Proc. 2006 Int. Symp. Advanced Control of Chemical Processes, volume 6, 875-880. Brazil.

Thornhill, N.F. and Horch, A. (2006). Advances and new directions in plant-wide controller performance assessment. In Proc. 2006 Int. Symp. Advanced Control of Chemical Processes, volume 6, 29-36. Brazil.

$\mathrm{Yu}$, J. and Qin, S.J. (2008). Statistical mimo controller performance monitoring, part ii: Performance diagnosis. J. Process Control, 18(3-4), 297-319.

Yue, H.H. and Qin, S. (2001). Reconstruction-based fault identification using a combined index. Industrial \&6 Engineering Chemistry Research, 40(20), 4403-4414.

Zhang, Q. and Li, S.Y. (2006). Performance monitoring and diagnosis of multivariable model predictive control using statistical analysis. Chinese J. Chem. Eng., 14(2), 207-215.

Zhao, C., Zhao, Y., Su, H.Y., and Huang, B. (2009). Economic performance assessment of advanced process control with lqg benchmarking. J. Process Control, 19(4), 557-569.

Zhao, Y., Gu, Y., Su, H.Y., and Huang, B. (2008). Extended prediction error approach for mpc performance monitoring and industrial applications. In Proc. 17th IFAC World Congress, volume 17, 14894-14899. Korea. 